\title{
BMJ Open Smartphone application for preventing depression: study protocol for a workplace randomised controlled trial
}

Mark Deady, ${ }^{1}$ David A Johnston, ${ }^{1}$ Nick Glozier, ${ }^{2}$ David Milne, ${ }^{3,4}$ Isabella Choi, ${ }^{2}$ Andrew Mackinnon, ${ }^{1}$ Arnstein Mykletun, ${ }^{5}$ Rafael A Calvo, ${ }^{3}$ Aimee Gayed, ${ }^{6}$ Richard Bryant, ${ }^{7}$ Helen Christensen, ${ }^{1}$ Samuel B Harvey ${ }^{1}$

To cite: Deady M, Johnston DA, Glozier N, et al. Smartphone application for preventing depression: study protocol for a workplace randomised controlled trial. BMJ Open 2018;8:e20510. doi:10.1136/ bmjopen-2017-020510

- Prepublication history for this paper is available online. To view these files, please visit the journal online ( $h t t p: / / d x . d o i$. org/10.1136/bmjopen-2017020510).

Received 7 November 2017

Revised 6 June 2018

Accepted 7 June 2018

Check for updates

(c) Author(s) (or their employer(s)) 2018. Re-use permitted under CC BY-NC. No commercial re-use. See rights and permissions. Published by BMJ.

${ }^{1}$ Black Dog Institute, Faculty of Medicine, University of New South Wales, Sydney, New South Wales, Australia

${ }^{2}$ Brain and Mind Centre, University of Sydney, Sydney, New South Wales, Australia

${ }^{3}$ School of Electrical and Information Engineering, University of Sydney, Sydney, New South Wales, Australia ${ }^{4}$ School of Systems

Management and Leadership, University of Technology Sydney, Sydney, New South Wales,

Australia

${ }^{5}$ Norwegian Institute of Public Health, Oslo, Norway

${ }^{6}$ School of Psychiatry, UNSW,

Sydney, NSW, Australia

${ }^{7}$ School of Psychology, UNSW,

Sydney, NSW, Australia

Correspondence to

Dr Mark Deady;

m.deady@unsw.edu.au

\section{ABSTRACT}

Introduction Depression is the leading cause of life years lost due to disability. Appropriate prevention has the potential to reduce the incidence of new cases of depression, however, traditional prevention approaches face significant scalability issues. Prevention programmes delivered by via smartphone applications provide a potential solution. The workplace is an ideal setting to roll out this form of intervention, particularly among industries that are unlikely to access traditional health initiatives and whose workplace characteristics create accessibility and portability issues. The study aims to evaluate the effectiveness of a smartphone application designed to prevent depression and improve well-being. The effectiveness of the app as a universal, selective and indicated prevention tool will also be evaluated.

Methods and analysis A multicentre randomised controlled trial, to determine the effectiveness of the intervention compared with an active mood monitoring control in reducing depressive symptoms (primary outcome) and the prevalence of depression at 3 months, with secondary outcomes assessing well-being and work performance. Employees from a range of industries will be invited to participate. Participants with likely current depression at baseline will be excluded. Following baseline assessment, participants, blinded to their allocation, will be randomised to receive one of two versions of the application: headgear (a 30-day mental health intervention) or a control application (mood monitoring for 30 days). Both versions of the app contain a risk calculator to provide a measure of future risk. Analyses will be conducted within an intention-to-treat framework using mixed modelling, with additional analyses conducted to compare the moderating effect of baseline risk level and depression symptom severity on the intervention's effectiveness.

Ethics and dissemination The current trial has received ethics approval from the University of New South Wales Human Research Ethics Committee (HC17021). Study results will be disseminated through peer-reviewed journals and conferences.

Trial registration number ACTRN12617000548336; Results.

\section{INTRODUCTION}

Mental health (MH) conditions are the leading cause of long-term disability in most middle-income and high-income countries. ${ }^{12}$
Strengths and limitations of this study

Large multisite trial (over 2000 participants), making this the largest ever depression prevention trial.

- Intention-to-treat analysis and randomised controlled trial gold standard design, with active control.

- Ability to compare the relative efficacy of universal, selective and indicated prevention approaches.

- The use of a novel app-based intervention in the workplace.

This is mainly due to the impact of anxiety and mood disorders in individuals of working age, which have pooled 12-month prevalence rates of $10.6 \%$ and $4.1 \%$, respectively. ${ }^{3-5}$ To date, most of the effort to reduce the burden of these disorders has been targeted at ensuring treatment is given to those with manifest disorders. Although effective treatments are available, cost-effectiveness models suggest that even in the unlikely event of optimal treatment being delivered to all cases, only $35 \%-50 \%$ of the overall burden of common mental disorders would be alleviated. ${ }^{6}$ As a result, effective preventative interventions, that intervene prior to onset of full-diagnostic disorder, must be a key component of the public health response to this problem. ${ }^{78}$

There is increasing evidence that prevention of mental disorders is possible, either via programmes based on reducing established, modifiable risk factors (including transdiagnostic factors) or by training to enhance resilience. ${ }^{9}$ However, the cost associated with delivering most face-to-face psychological prevention programmes has made large scale roll-outs unfeasible. ${ }^{10}$ Consequently, most trials of MH prevention programmes have either used youth samples, where teachers and classrooms can be used to deliver faceto-face interventions, ${ }^{11-13}$ or adult populations with subclinical symptom levels ${ }^{14} 15$ or other comorbid conditions placing them 
at substantially increased risk. ${ }^{16}$ As a result, it remains unclear which, if any, preventive interventions are effective in a more generalised population. A recent systematic review and meta-analysis suggested that there is emerging evidence that several common psychotherapeutic techniques, such as cognitive- behavioural therapy (CBT), may be effective in preventing common mental disorders in non-selected general populations. ${ }^{17}$ To date though, large-scale studies are lacking, and the relative utility of some of the less complex (and cheaper) techniques, such as behavioural activation (BA), remains unknown. ${ }^{18}$ There is also a paucity of evidence indicating which populations might be the prime targets for prevention efforts. Prevention efforts are generally categorised into three groups: universal, where a prevention initiative is directed at an entire population; selective, where efforts are focused on those at high risk and indicated, where prevention is directed at those with emerging symptoms.

As the workplace is a dominant setting in the lives of many adults, it is increasingly being recognised as a prime location for $\mathrm{MH}$ prevention interventions, ${ }^{19}$ especially as $44 \%$ of working Australians report workplace issues as a source of stress. ${ }^{20}$ While workplace stress is not localised to any particular industry, the issue appears to be more pertinent in male-dominated industries (MDIs) where employees have been found to have elevated rates of anxiety and mood disorders compared with other industries. $^{21} 22$ MDIs highlight the interplay between workplace-related factors (highly mobile, remote or isolated, irregular workloads ${ }^{21}$ ) and the sociodemographic features of the worker (lower health literacy and lower rates of help seeking ${ }^{2324}$ ) in contributing to these elevated rates. These industries also highlight the current limitations of conventional prevention programmes, which are neither accessible nor portable to all industries and represent a traditionally underserviced group. Recent rapid growth in the areas of eHealth and mHealth (healthcare practices supported by internet or mobile phone technologies) represent new frontiers for delivering and targeting MH interventions. ${ }^{25-29}$ These developments provide a potential solution to the barriers involved in delivering individually tailored prevention measures across a wide population in a practical, anonymous (thereby counteracting MH stigma) and cost-effective manner. Indeed, the ubiquity of personally owned devices increases the feasibility of mobile $\mathrm{MH}$ care interventions. ${ }^{30}$ While the effectiveness of internet and smartphone applications as a treatment for common mental disorders has been evaluated extensively ${ }^{31}$-and the workplace as a specific setting for these interventions- to $^{19}$ date there have been relatively few trials examining the effectiveness of these types of intervention in preventing mental disorders. ${ }^{32}$

The proposed headgear trial aims to evaluate the effectiveness of a new smartphone app designed to prevent depression in Australian workers. As well as assessing the overall effectiveness of the intervention, the study also aims to compare the relative efficacy of universal, selective and indicated prevention approaches as defined through the identification of relevant subgroups of the sample recruited.

\section{METHODS \\ Design}

The above aims will be achieved in a multicentre randomised controlled trial, with two parallel arms. Two smartphone app-based interventions will be compared: a new intervention for primary prevention of common mental disorders (headgear) and a specially developed active control. Assessments will occur at baseline, postintervention (5 weeks) and 3-month follow-up. The study is registered with the Australian New Zealand Clinical Trials Registry (ACTRN12617000548336). Consent will be obtained electronically from all participants and any identifiable data encrypted. The study will be conducted in accordance with the Declaration of Helsinki ${ }^{33}$ and is compliant with the Standard Protocol Items for Reporting in Trials ${ }^{34}$ and Consolidated Standards of Reporting Trials ${ }^{35}$ guidelines.

\section{Setting and participants}

The study will recruit Australians who are currently employed and will sample more selectively from a range of MDIs. A MDI is defined as one in which $\geq 70 \%$ of workers are male. ${ }^{36}$ In Australia, these industries include agriculture/forestry/fishing, utility services (electricity, gas, water and waste), wholesale trade, manufacturing, transport/postal/warehousing, mining and construction. ${ }^{36}$ Emergency services and defence also fit this definition, but were not considered unique industries by the Australian Bureau of Statistics (ABS), for this study they were considered as such.

A number of industry partner organisations will provide the trial sites for this study. These partners comprise a range of industries including transport, postal, mining, construction, emergency services and utility services and have sites across Australia. Each organisation has offered access to specific sites, or to their entire workforce.

We will aim to recruit a total sample of at least 2100 employed adults across Australia (see sample size calculation below). Industry partner organisations will promote the study via their respective health and well-being officers, chaplaincy and peer-support committees, along with email and newsletter advertisements. Social media advertising targeted at employed people will also be used to recruit beyond the partner organisations using a similar approach to previous studies. ${ }^{37}$ The study will also be promoted via members of the research team presenting at partner worksites. Both men and women will be recruited, though it is anticipated that more than half of the sample will be men due to the focus on MDIs.

\section{Eligibility criteria}

Initial eligibility criteria are: having a valid telephone number, ownership of an Apple-operating/Android-operating smartphone, currently employed and living in 
Australia. Participants will be excluded if they do not have reliable internet access at home or at work, or cannot read English. As this trial is focused on the prevention of depression, participants will also be excluded from this trial (although still permitted to use the app) if they have substantial levels of depression symptoms at baseline, as indicated by a score above 14 or meeting the major depressive disorder (MDD) diagnosis algorithm using the Patient Health Questionnaire-9 item (PHQ-9).

\section{Interventions}

Active intervention: headgear

The intervention condition, headgear, is a smartphone application-based intervention centred on BA and mindfulness. BA is a therapy that reconnects people to an environment of positive reinforcement using value-driven goal setting, ${ }^{3839}$ and has been shown to perform as well as full $\mathrm{CBT}^{18}$ for the treatment of depression. Although primarily used as a treatment for clinically depressed clients, a recent metanalytic review has found a significant and moderately sized effect of BA interventions on well-being (a risk factor for depression ${ }^{40}$ ), regardless of depression status. ${ }^{41}$ The other component, mindfulness is both a process (meditation) and an outcome (mindful awareness), ${ }^{42}$ shown to have diagnosis independent effects in reducing depression, anxiety ${ }^{43}$ and stress levels (a risk factor for depression ${ }^{44}$ ) in healthy individuals. ${ }^{45}$

The main therapeutic component of the headgear app takes the form of a 30-day intervention in which users complete one 'challenge' daily (5-10 min per day). These 'challenges' feature a variety of evidence-based therapeutic techniques and formats. The modules are structured, so a participant might be presented with a psychoeducational video (eg, on BA, mindfulness, coping skills or resilience) to promote understanding of the concept and/or skill on 1 day and then on a following challenge day, participants would then practice the skill or apply a concept that was previously learnt. Examples of practical exercises include mindfulness exercises, value-driven activity planning, goal setting and coping skill development (problem solving, sleep, grounding, alcohol use, assertiveness and training in adaptive forms of coping).

For BA, participants will be encouraged to consider how increased engagement in activities that are valued increases the chance of deriving pleasure and feeling a sense of achievement from life. BA's theoretical underpinning is that a person's development and maintenance of depression is the result of avoidant behaviour as a coping mechanism to an environment characterised by low levels of positive reinforcement and/or high levels of aversive control. ${ }^{46}$ Thus, by targeting the avoidance behaviours as well as broadening the repertoire of behaviours an individual engages in, people are more likely to experience positive reinforcement in their environment. During mindfulness exercises, participants will be taught how they can pay more attention to and be more accepting of momentary experiences, through standard meditation practices such as mindfulness of breath, body scan and cognitive defusion. Through such contemplative practices, the quality of an open, accepting mind is cultivated. Such practices are in direct contrast to the maladaptive habitual mental processes of depressive disorders which have a propensity towards an avoidance of experience and an intolerance of negative emotions and cognitions. ${ }^{47} 48$ Both BA and mindfulness have been shown to be some of the most popular techniques among workers in MDIs. ${ }^{49}$

Prior to completing the intervention users will complete a risk calculator, which will assess and provide participants with personalised feedback regarding their risk for future $\mathrm{MH}$ issues. The risk calculator is an algorithm consisting of 20 items developed from the Household, Income and Labour Dynamics in Australia Survey (HILDA), and has been validated in the Australian adult population. ${ }^{50}$ The HILDA questions and response items were replicated from the original items included in the HILDA survey, apart from age, which is measured here as a continuous measure. Users will receive personalised risk feedback immediately after completing the risk calculator. The personalised risk feedback involves an interactive icon array which displays the calculated numerical risk estimate of developing anxiety and depression within the next year, along with a text description.

Other components of the headgear intervention app include interactive mood monitoring, a toolbox of skills (which is built as the intervention is completed) and technical support service helplines. The application has been designed to maximise user engagement and adherence, incorporating reward acquisition on completion of each daily challenge. The application is the result of an iterative development process to allow for optimal functionality and design ${ }^{49}$ Consideration was also given to the context and population of working men and women in which the trial is taking place. As the majority of the population are not currently experiencing distressing symptoms of mental ill health, engagement-both generating motivation to engage and maintaining user engagement over time-is important. To this end steps have been taken to promote user motivation and engagement via the 'challenge' concept in conjunction to other related components and technical features aimed to increase the app's appeal. ${ }^{52-54}$

\section{Control condition: mood monitoring smartphone application}

The control condition is a smartphone application that, to the user, will have the same name and a virtually identical look and 'feel' as headgear. However, there is no skill development and no components of BA or mindfulness therapy. To control for the other components of the headgear application, the control condition will encourage users to use the inbuilt mood monitor daily over a 30-day period. To ensure consistent approaches between the two applications, participants in the control group will receive daily reminders to record their moods via the control smartphone application. In conjunction to this, participants are able to review their 'mood history' in a calendar that displays the mood recorded across each 
day. Participants will also have access to the risk calculator that will provide participants with individualised risk feedback. Controlling for mood monitoring, which is a common feature of many commercially available smartphone applications, is essential as this alone may bring awareness to an individual's mood and allow them to gain greater understanding of the causative behaviours related to their mood and associated improvements in mood.

\section{Procedure}

All interested employees will be directed to their respective app store (iTunes or Google Play) directly via advertisements or via a dedicated website (http:/ / headgear.org. $\mathrm{au}$ ). On downloading the app, participants will provide informed consent and then undergo initial screening. Participants who meet the inclusion criteria will then be randomised to receive either the full headgear app or the attention-matched control version of the app. Participants will be blinded to their allocation.

Those scoring above 14 on the PHQ-9 (or meeting MDD diagnosis using the PHQ-9 algorithm ${ }^{55}$ at baseline will be provided with appropriate referral information to health services and crisis lines and will be encouraged to seek help from their general practitioner (GP). This group will be excluded from this trial. The flow of participants through the study phases is shown in figure 1 .

\section{Patient and public involvement}

As this is a prevention study, the participants will be employed members of the general public, rather than patients. End users were involved in the design of the intervention used in the study through participatory workshops ${ }^{51}$ and separate quantitative surveys, ${ }^{49}$ reported on previously. The findings of these studies directly

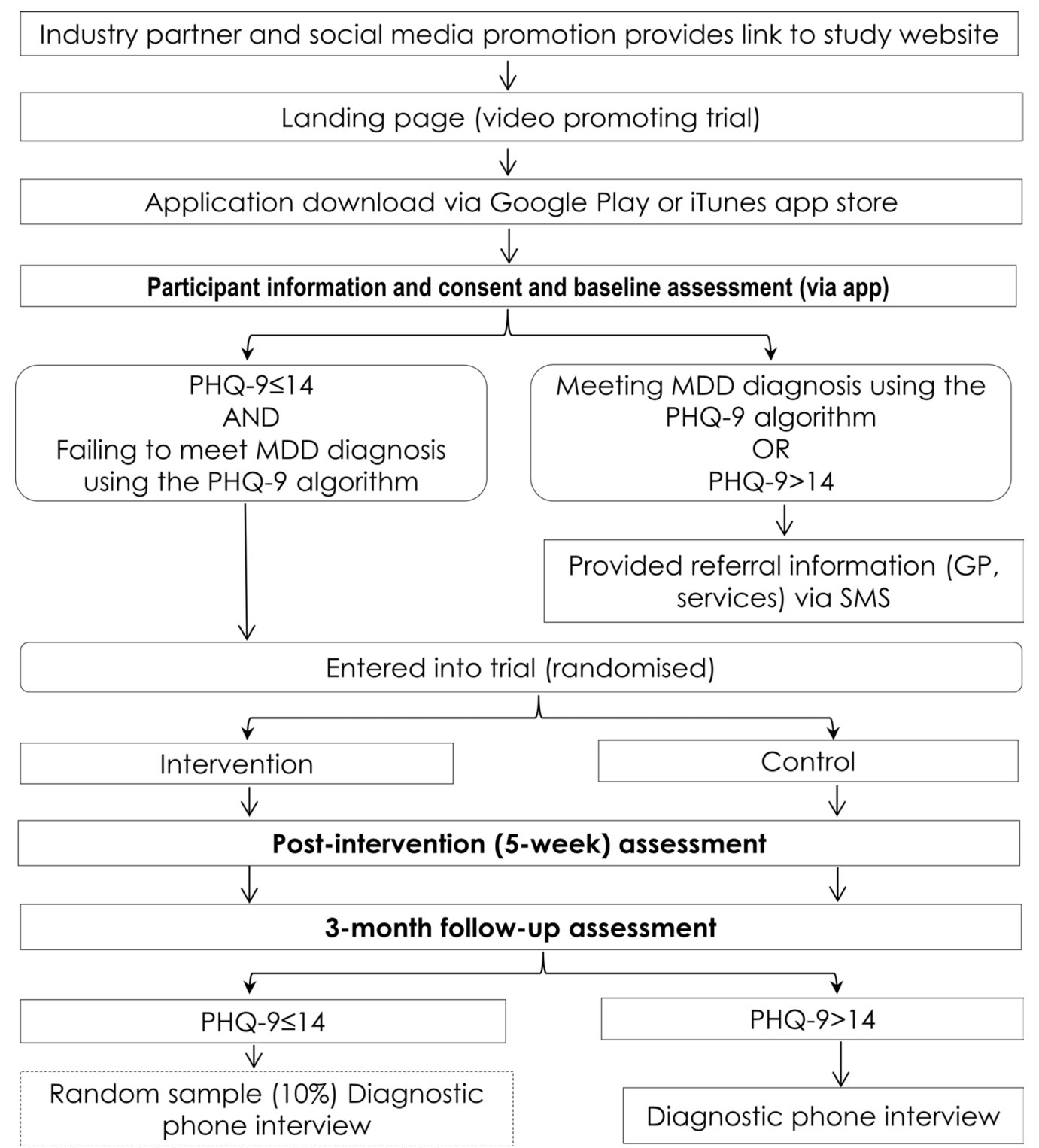

Figure 1 Flow of participants through the trial. GP, general practitioner; MDD, major depressive disorder; PHQ-9, Patient Health Questionnaire-9 item. 
informed the research question and outcome direction of this trial. Dissemination of results to participants will occur via the project website. All participant burden of the trial will be clearly laid out in the information sheet prior to consent.

\section{Random allocation}

Immediately following the completion of the baseline questionnaire, the smartphone application will communicate with a central server (responsible for collecting all study data) to assign the participant to either the control or treatment condition. The randomisation algorithm used by the server will follow a block design with a block size of 10, to ensure an equal number of participants are assigned to each condition.

\section{Administration of assessments}

Assessments will be completed at baseline, postintervention ( 5 weeks) and 3-month follow-up. Baseline assessment includes outcome measures pertaining to depression symptomatology PHQ-9, ${ }^{56}$ well-being (WHO-5 Well-Being Index (WHO-5) ${ }^{57}$ ), anxiety symptomatology (as measured by the General Anxiety Disorder-2 item; GAD-2 ${ }^{59}$ ), resilience (Connor-Davidson Resilience Scale 10-item; CD-RISC $10^{60}$ ), work performance and absenteeism (Health and Work Performance Questionnaire; $\mathrm{HPQ}^{61}$ ), demographic information and service use/management. The application monitors usage data including time (amount of time spent in-app) and frequency of use, number of log-ins and activity completion rates. This data will be used to examine programme engagement.

Postintervention assessment will occur at 5 weeks postbaseline, to allow users one extra week to complete the 30-day programme. To address the possibility that users may delete the app during the course of the trial, which may hamper follow-up attempts, later follow-ups will occur via phone numbers provided, rather than via the application. Participants will complete an online questionnaire similar to that at baseline (see table 1). This will be replicated at 3 months postbaseline. Participants will receive up to three phone-based reminders to complete each follow-up. Follow-up measures will be accepted provided they are obtained within a 90-day range of the planned assessment (up to 90 days after scheduled assessment). On completion of each follow-up assessment, participants will be entered into a draw for one of four $\$ 200$ Visa gift cards. Strategies to increase response rates followed previous published guidance ${ }^{62}$ including; reducing the length of follow-up questionnaires and giving participants feedback on their progress while filling out the questionnaire. Similarly, each phone-based reminder incorporated a reminder of the gift coupon to facilitate increased motivation to complete the questionnaires.

\section{Specific measures used in online assessments}

The PHQ-9 will be used to measure depression symptoms. ${ }^{63}$ The PHQ-9 is a reliable and valid nine-item measure of depression severity over the past 2 weeks in clinical and general population samples. ${ }^{564-66}$ Each of the nine items of the PHQ-9 is scored as 0 (not at all), 1 (several days), 2 (more than half the days) or 3 (nearly every day). As a screening tool, summing the nine items, (score range 0-27 with 0 indicating no depressive symptoms and 27 indicating all symptoms occurring nearly daily). The criterion and construct validity of the PHQ-9 have previously been demonstrated, with $73 \%$ sensitivity and $98 \%$ specificity (using the depression diagnostic algorithm) in detecting major depression compared with clinician-based assessment ${ }^{6367}$ and, regardless of diagnostic status, typically represents clinically significant depression. ${ }^{63}$ The measure has demonstrated excellent internal consistency (Cronbach's $\alpha>0.85$ in multiple samples) and test-retest reliability of $0.84 .^{55}$

Anxiety will be measured using the GAD-2. ${ }^{59}$ The GAD-2 consists of the two core criteria for GAD, which have also been shown to be effective screening items for panic, social anxiety and post-traumatic stress disorders. ${ }^{59}$ This measure has also been validated in the general population. ${ }^{68}$ Equivalent to the parent scales, the GAD-2 begins with the stem question: 'Over the last 2 weeks, how often have you been bothered by the following problems?'

Table 1 Assessment measures

\begin{tabular}{|c|c|c|c|}
\hline & Baseline & Postintervention & 3-month follow-up \\
\hline Demographics & $\times$ & & \\
\hline Patient Health Questionnaire-9 item (PHQ-9) ${ }^{55} 56$ & $\times$ & $\times$ & $x$ \\
\hline WHO-5 Well-Being Index 5758 & $\times$ & $x$ & $x$ \\
\hline Connor-Davidson Resilience Scale 10-item ${ }^{69}$ & $\times$ & $\times$ & $\times$ \\
\hline Mini-International Neuropsychiatric Interview ${ }^{72 \star}$ & & & $\times$ \\
\hline Service utilisation and management items & $x$ & $\times$ & $\times$ \\
\hline Programme feedback & & $x$ & \\
\hline
\end{tabular}

*Only for those scoring above $14 \%$ and $10 \%$ of those scoring below 14 on the PHQ-9 at 3-month follow-up will be contacted via telephone. 
Response options are 'not at all', 'several days', 'more than half the days' and 'nearly every day', scored as 0 , 1,2 and 3 , respectively (total ranging from 0 to 6 ). Scale scores of $\geq 3$ are suggested as cut-off points between the normal range and probable cases of anxiety. ${ }^{59}$

Resilience will be measured by the CD-RISC, a 10-item self-report scale demonstrated to be psychometrically sound with high internal consistency (Cronbach's $\alpha=0.89$ ), construct validity and test-retest reliability in the general population and in clinical settings. ${ }^{60}$ Total scores range from 0 to 40 with higher scores corresponding to greater resilience. Validity is high relative to other measures and reflects differentiation in resilience among diverse populations, showing that higher levels of resilience are consistent with lower levels of perceived stress vulnerability. ${ }^{60}$ The CD-RISC has been shown to differentiate between individuals who function well after adversity from those who do not and measures the core features of resilience and the ability to tolerate experiences. ${ }^{69} \mathrm{It}$ is believed that increased resilience may reduce rates of mental ill health. ${ }^{70}$

Well-being will be assessed using the 5 -item WHO- $5 .{ }^{57} 58$ Raw scores range from 0 to 25 where 0 indicates the worst possible quality of life while a score of 25 represents the best possible quality of life. A score $\leq 13$ or an answer of 0 or 1 on any of the five items shows poor well-being. WHO-5 is a psychometrically sound measure of well-being with high internal consistency (Cronbach's $\alpha=0.84$ ) and convergent associations with other measures of well-being. ${ }^{71}$

Work performance will be measured using three items (performance items A10, A11, A12) from the HPQ ${ }^{61}$ and two additional items pertaining to past month sickness absence (days absent, days absent for MH reasons) and past 6-month week-long sickness absence (weeks absent, weeks absent for MH reasons).

Service use and management items comprised seven items assessing lifetime and past month service use, along with current medication use. Participants were also asked about their abilities (perceived capability and effectiveness) to manage their mental fitness, and autonomy (choice and freedom) in management. These were scored on a 7-point Likert scale from strongly disagree to strongly agree.

\section{Diagnostic interview}

All those scoring 14 and above and a 10\% random sample of those scoring below 14 on the PHQ-9 at 3-month follow-up will be contacted via telephone. Registered psychologists, trained in the use of the diagnostic instrument and blinded to randomisation, will then conduct a telephone-based diagnostic interview using the 'current' depression section of the Mini-International Neuropsychiatric Interview (MINI). ${ }^{72}$ The MINI has shown to be reliable and valid in eliciting symptom criteria used in the Diagnostic and Statistical Manual of Mental Disorders, Fourth Edition, Text Revision (DSM-IV-R) and International Statistical Classification of Diseases and Related Health Problems 10th Revision (ICD-10). The scale also has good concordance with the Composite International Diagnostic Interview with specificity ranges of $0.72-0.97$ for non-psychotic disorders in psychiatric and non-psychiatric populations. ${ }^{72}$

\section{Safety protocol}

In any trial concerned with $\mathrm{MH}$, there is the potential for psychological distress in participants. Although this study is not targeting unwell populations, based on prevalence data, it is predicted that $4.5 \%$ will score above 14 on the PHQ-9 (or meet diagnosis using the instrument algorithm) at follow-up. ${ }^{73}$ As this unwell group may have more motivation to participate, we might conservatively predict this to be higher (15\%). Those falling into this category (or reporting suicidal ideation) either within the app or at follow-up will trigger the user to be directed to a 'get support' page (at each assessment point) and will suggest the participant seek further help from these support services or their GP. Additionally, an optional call-back service for individuals requiring further support or direction is provided. This callback will occur within 4 days from a member of staff with training in $\mathrm{MH}$ care (psychologist) and will guide participants into necessary care arrangements. If the team member still has concerns for the participant's safety, an accredited psychiatrist will make contact with the participant as soon as possible. These participants will also receive an Short Messaging Service (SMS) with a range of support service contacts with reiteration of the advice to consult with their GP regarding their $\mathrm{MH}$.

\section{Study hypotheses and outcomes}

We hypothesise that participants receiving the headgear intervention will have lower levels of depression symptomatology at postintervention and 3-month follow-up, compared with participants in the attention-matched control condition. While the primary analyses will be conducted on the entire sample (to examine the universal intervention effect), we also predict the intervention effect to vary according to risk level (based on the risk calculator) and level of depression symptoms at baseline. ${ }^{74}$ As such, analyses will be conducted to compare the relative effectiveness of the headgear intervention based on participants' level of risk and depression symptoms at baseline.

Second, it is hypothesised that relative to control, headgear participants will have lower rates of depressive disorder as detected by the PHQ algorithm and the two-stage diagnostic process outlined above. The intervention group is also hypothesised to have improved anxiety symptomatology, well-being, resilience and work performance at postintervention and follow-up relative to controls.

\section{Primary outcome}

The primary outcome will be the level of depressive symptomatology (as measured by the PHQ-9 sum score). The study primary endpoint will be the 3-month follow-up. 


\section{Secondary outcomes}

A range of secondary outcomes will be considered including change in anxiety symptoms (as measured by the GAD-2) at both 5-week and 3-month follow-up. Similarly, incident cases of depression at 5-week and 3-month follow-up (as measured by diagnostic interview and the PHQ-9 diagnostic algorithm) and a reduction in the estimated risk of depression (as measured by the depression risk algorithm) at 3-month follow-up are also secondary outcomes. Finally, change in Well-being (as measured by the WHO-5), resilience (CD-RISC 10), service use, self-determination (4-item scale mentioned above) and occupational functioning (as measured by the HPQ and sickness absence questions) at both 5-week and 3-month follow-up will be outcomes of interest.

\section{Statistical analysis}

Analysis plan

The composition of our sample will be demonstrated via simple descriptive statistics of participants' age, gender, job role, industry group and baseline $\mathrm{MH}$ measures. Random imbalances between the intervention and control group will be examined for using $\chi^{2}$ tests. Primary analyses will be undertaken on an intent-to-treat basis, including all participants as randomised, regardless of treatment received or withdrawal from the study, with analysis considering recruitment strategy. Mixed-model repeated measures (MMRM) models will be used to analyse change in the primary outcome measure (PHQ9 ). The model used to test the primary hypothesis will only include intervention allocation and occasion of measurement as 'predictors' in the MMRM analysis of variance. Mathematical transformation or categorisation of raw scores will be undertaken to meet distributional assumptions and address any violation of assumptions required in MMRM models. When transformations have been undertaken to better meet distribution assumptions, models using the transformed data will be considered the main test of the primary hypotheses. MMRM uses all available data and does not involve any substitution of missing values with supposed or estimated values. The assumptions underlying MMRM allow 'missingness' to be related to observed variables in the analysis but not to unobserved values (termed 'missing at random'). An a priori planned comparison of change from baseline across the 3-month follow-up period will be used to test the primary hypothesis. An unstructured variance-covariance matrix will be used to accommodate relationships between observations at different occasions. Variables found to be substantially imbalanced between groups postrandomisation will be included in sensitivity analysis to explore the robustness of any findings to any chance imbalance. Similar analyses of scaled secondary measures will assess differential change due to intervention arm. For dichotomous outcomes such as caseness, a comparable generalised mixed modelling approach will be used. Relative and reduction in risk of depression based on PHQ-9 score status and MINI interviews will be estimated at the trial endpoint and follow-up. Number needed to treat will be derived from these values. All analyses will use two-sided tests, with an alpha value set at 0.05 .

Baseline characteristics will be used to define subgroups that would be the targeted if the apps were offered as a selective or indicated prevention. Group membership will be used models to evaluate moderation of effectiveness by adding appropriate interaction terms and undertaking planned comparisons. The effect on outcome of level of baseline depressive symptom levels, recruitment method and risk of depression calculated at baseline will be explored using an analysis of covariance approach using baseline measures as a covariate and including a covariate by intervention arm interaction term in models. The effectiveness of the active intervention at clinically relevant levels of baseline covariates will be assessed using planning comparisons while the lowest values of covariates associated with a significant benefit of the intervention will be established using a Johnson and Neyman ${ }^{75}$ approach.

\section{Sample size}

As a universal prevention intervention, the size of the effect of the intervention is anticipated to be relatively small. Meta-analysis of previous trials of workplace prevention of depression showed a small effect size of $\mathrm{d}=0.17{ }^{76}$ Similarly, unsupported internet-based treatment of depression has been shown to have a small effect size of $d=0.25,{ }^{77}$ consistent with the findings from a meta-analysis published more recently. ${ }^{78}$ Power calculations were carried out using the R package simR, which can undertake power calculations for mixed models such as those proposed to use in this study using simulation techniques. ${ }^{79}$ Power was set at $80 \%$, alpha at 0.05 , with a two-sided hypothesis test and an assumption of correlation at 0.50 between preintervention and postintervention scores. This estimated a sample size of 1134 is required to detect an effect size of $d=0.20$ on the total PHQ-9 scale score at 3-month follow-up. It is predicted that $10 \%$ of the population will score above the exclusion PHQ-9 cut-off at baseline, ${ }^{73}$ increasing the initial sample required by 126 . Finally, using a conservative attrition rate of $40 \%$, an initial total sample of 2100 will need to be recruited and randomised.

\section{Dissemination}

We anticipate the study will be completed by 1 December 2017. Results of this study will be disseminated for publication in peer-reviewed journals and key findings presented at national and international conferences.

\section{DISCUSSION}

This study will be the first randomised controlled trial to examine the effectiveness of a MH smartphone app in preventing common mental disorders. It will also be the largest MH prevention trial to ever be undertaken in a workplace setting. Given the rising cost of mental illness 
to employers across the developed world, ${ }^{2}$ the fact that workplaces are one of the dominant settings in the lives of most individuals, and the increasing awareness of the role work and workplaces can play in the development of mental illness ${ }^{80}$ the movement of prevention MH interventions into the workplace is arguably long overdue.

This study will provide valuable evidence regarding the utility of mHealth tools as a prevention method. Such applications provide a way to deliver interventions across a wide population due to flexibility and portability, increased user retention, tracking and monitoring features, and personalised feedback and support. ${ }^{26} 81$ However, little is known of their utility for prevention. The size and design of this study will also allow for the direct comparing of effects of a single intervention at universal, selective and indicated levels of risk. This is a unique aspect of the study.

To date, much of the research examining prevention interventions for depression have focused on CBT-based techniques. Recent evidence suggests mindfulness-based interventions may have particular utility in workplace-based eHealth interventions. ${ }^{32}$ Similarly, in recent years there has been renewed interest in behavioural treatment approaches for mental disorders, particularly for depression ${ }^{39} 82$ and growing evidence of the effectiveness of such interventions ${ }^{18}$ and some interest in adapting these techniques for use with mHealth technologies. ${ }^{83}$ There is also recent work suggesting individuals working in some traditionally undersampled professions may prefer behavioural techniques over other therapeutic techniques that focus on cognitions or emotions. ${ }^{49}$ Other studies have suggested men may prefer more directive approaches to psychological therapy ${ }^{84}$ and therapy models that emphasise action, ${ }^{85}$ which again would be in keeping with behavioural activity and mindfulness-based techniques. Given these findings, direct testing of these types of techniques, particularly among men, is urgently needed.

There are, however, some limitations in the proposed trial. First, since the intervention is unguided and delivered via a smartphone application, trial attrition and disengagement are potential issues. ${ }^{86} 87$ Reasons for attrition are complex and in reality, there are contradictory possibilities of drop-out due to dissatisfaction or lack of engagement, as opposed to drop-out due to a sense that the individual feels their needs have been met. ${ }^{88}$ Ultimately, use of a conservative attrition rate and statistical methods robust to data missing at random, it is hoped this limitation will be minimised. A related issue is the expected challenges of collecting follow-up data within the required time frame. It is expected that many participants will be delayed in returning their 3-month follow-up data. As noted above, we have allowed a relatively wide window for follow-up timing (90 days), which should help increase follow-up numbers but which at the cost of making it harder to interpret the impact of treatment over set time periods. As with any controlled trial, the possibility of contamination exists, where participants randomised to different conditions but working in the same firm may exchange information. However, given the substantial sizes of the organisations involved, the stigma associated with $\mathrm{MH}$ and the fact the smartphone application is specifically designed to allow participants to work though modules alone, it is anticipated that this occurrence is unlikely. Another limitation related to the use of a smartphone application is the potential that technological issues, such as reduced network coverage in some areas, and a rapidly changing market may create barriers, particularly among certain industries.

Participants will be recruited through industry partners on a volunteer basis. This will inevitably create a sample that is not necessarily generalisable to the whole working population, however, this will be somewhat ameliorated through social media recruitment targeting people who are employed. While the application is designed with specific working populations from MDIs as its target, the content of the application should have a general relevance to any working individual. Additionally, voluntary self-selection makes the concept of universal prevention more contentious. Due to the opt-in nature of research trials and the structure of $\mathrm{MH}$ prevention programmes themselves, this is an issue faced by all trials of this nature and is likely to reflect the reality of such programmes outside of a research setting. Another potential issue relates to testing the effectiveness of headgear as an indicated prevention tool. To do this, participants scoring in the diagnostic "grey zone ${ }^{89}$ on the PHQ-9 will be included. This will be done to ensure that people with subsyndromal depression are included in the trial, as they would be in a 'real-world' roll-out of any prevention initiative. It is acknowledged, however, that this approach may result in some cases of mild depression being misclassified as subsyndromal, resulting in a potential conflating of the concepts of prevention and very early treatment.

In attempting to increase the app's anonymity (due to MH stigma) and removal of barriers, no diagnostic interview will occur at baseline, as such initial diagnosis will not be verified. The research team felt this was a necessary trade-off with respect to the case detection while also concurrently allowing for large numbers of participants to be recruited economically with regard to time and resources. ${ }^{9-92}$ Using the dual methods of excluding those both scoring above 14 (sensitivity $=68 \%$, specificity $=95 \%{ }^{55}$ ) and meeting the algorithm's criteria, we believe we can be confident the baseline sample will be free of any significant depressive illness. The two-phase design ${ }^{94}$ for follow-up interviews will allow us to then examine incident cases of depression as a secondary outcome.

Overall, outcomes of the headgear trial capacity to empower individuals and thereby dramatically enhance the way working adults manage their $\mathrm{MH}$ and well-being. By using evidence-based mHealth technologies, many of the feasibility, access and help-seeking obstacles often encountered with more traditional face-to-face interventions in this area can be overcome, allowing for maximal 
reach and population impact via prevention of common mental disorders.

Acknowledgements The authors would like to acknowledge and thank all the study participants who have helped shape the content of the intervention.

Contributors MD had a primary role in conceptualisation, write up and editing of this manuscript. DAJ had a role in conceptualisation, write up and editing of this manuscript. NG had a role in conceptualisation and editing of this manuscript. DM had a role in technical development and editing of this manuscript. IC had a role in conceptualisation, write up and editing of this manuscript. AM had a role in statistical conceptualisation and editing of this manuscript. AM had a role in conceptualisation and editing of this manuscript. RAC had a role in write up and editing of this manuscript. AG had a role in write up and editing of this manuscript. $\mathrm{RB}$ had a role in write up and editing of this manuscript. $\mathrm{HC}$ had a role in write up and editing of this manuscript. SBH (Senior author) had a role in conceptualisation, write up and editing of this manuscript. All authors read and approved of the final manuscript.

Funding This study was developed in partnership with beyondblue with donations from the Movember Foundation. RAC is funded by an Australian Research Council Future Fellowship FT140100824. SBH and MD are supported by funding from the iCare Foundation and NSW Health.

Disclaimer This paper summarises independent research and the views expressed herein are those of the authors.

Competing interests All authors declared that this is a beyondblue-funded study. All researchers have remained independent from the funders in the completion and submission of this work. MD, DAJ, IC, NG, DM, RAC and SBH were involved in the development of the headgear application. The IP is jointly owned by MD, IC, NG, DM, RAC and SBH, however, the authors do not currently receive any financial gain from this IP.

\section{Patient consent Not required.}

Ethics approval The trial has received ethics approval from the University of New South Wales Human Research Ethics Committee (HC17021)

Provenance and peer review Not commissioned; externally peer reviewed.

Open access This is an open access article distributed in accordance with the Creative Commons Attribution Non Commercial (CC BY-NC 4.0) license, which permits others to distribute, remix, adapt, build upon this work non-commercially, and license their derivative works on different terms, provided the original work is properly cited, appropriate credit is given, any changes made indicated, and the use is non-commercial. See:@http://creativecommons.org/licenses/by-nc/4.0/.

\section{REFERENCES}

1. Murray CJ, Vos T, Lozano R, et al. Disability-adjusted life years (DALYs) for 291 diseases and injuries in 21 regions, 1990-2010: a systematic analysis for the Global Burden of Disease Study 2010. Lancet 2012;380:2197-223.

2. Harvey SB, Henderson M, Lelliott $P$, et al. Mental health and employment: much work still to be done. Br J Psychiatry 2009;194:201-3.

3. Demyttenaere K, Bruffaerts R, Posada-Villa J, et al. Prevalence, severity, and unmet need for treatment of mental disorders in the World Health Organization World Mental Health Surveys. JAMA 2004;291:2581-90.

4. Somers JM, Goldner EM, Waraich P, et al. Prevalence and incidence studies of anxiety disorders: a systematic review of the literature. Can J Psychiatry 2006;51:100-13.

5. Waraich P, Goldner EM, Somers JM, et al. Prevalence and incidence studies of mood disorders: a systematic review of the literature. Can J Psychiatry 2004;49:124-38.

6. Andrews G, Issakidis C, Sanderson K, et al. Utilising survey data to inform public policy: comparison of the cost-effectiveness of treatment of ten mental disorders. Br J Psychiatry 2004;184:526-33.

7. Hickie IB. Preventing depression: a challenge for the Australian community. Med J Aust 2002;177 Suppl:S85-6.

8. Berkels $\mathrm{H}$, et al. Mental health promotion and prevention strategies for coping with anxiety, depression and stress related disorders in Europe. Dortmund: European Commission, 2004.

9. Cuijpers P, Van Straten A, Smit F. Preventing the incidence of new cases of mental disorders: a meta-analytic review. J Nerv Ment Dis 2005;193:119-25.
10. Solomon D, Proudfoot J, Clarke J, et al. e-CBT (myCompass), Antidepressant Medication, and Face-to-Face Psychological Treatment for Depression in Australia: A Cost-Effectiveness Comparison. J Med Internet Res 2015;17:e255.

11. Barrett PM, Farrell LJ, Ollendick TH, et al. Long-term outcomes of an Australian universal prevention trial of anxiety and depression symptoms in children and youth: an evaluation of the friends program. J Clin Child Adolesc Psychol 2006;35:403-11.

12. Stice $\mathrm{E}$, Shaw $\mathrm{H}$, Bohon $\mathrm{C}$, et al. A meta-analytic review of depression prevention programs for children and adolescents: factors that predict magnitude of intervention effects. J Consult Clin Psychol 2009;77:486-503.

13. Gladstone TR, Beardslee WR. The prevention of depression in children and adolescents: a review. Can J Psychiatry 2009;54:212-21.

14. Cuijpers P, Smit F, van Straten A. Psychological treatments of subthreshold depression: a meta-analytic review. Acta Psychiatr Scand 2007;115:434-41.

15. Rasing SP, Creemers DH, Janssens JM, et al. Effectiveness of depression and anxiety prevention in adolescents with high familial risk: study protocol for a randomized controlled trial. BMC Psychiatry 2013;13:316.

16. Lustman PJ, Clouse RE, Nix BD, et al. Sertraline for prevention of depression recurrence in diabetes mellitus: a randomized, doubleblind, placebo-controlled trial. Arch Gen Psychiatry 2006;63:521-9.

17. Deady M, Choi I, Calvo RA, et al. eHealth interventions for the prevention of depression and anxiety in the general population: a systematic review and meta-analysis. BMC Psychiatry 2017; $17: 310$.

18. Richards DA, Ekers D, McMillan D, et al. Cost and Outcome of Behavioural Activation versus Cognitive Behavioural Therapy for Depression (COBRA): a randomised, controlled, non-inferiority trial. Lancet 2016;388:871-80.

19. Tan L, Wang MJ, Modini M, et al. Preventing the development of depression at work: a systematic review and meta-analysis of universal interventions in the workplace. BMC Med 2014;12:74

20. Casey L, Liang R. Stress and Wellbeing in Australia Survey. AP. Society 2014

21. Battams S, Roche AM, Fischer JA, et al. Workplace risk factors for anxiety and depression in male-dominated industries: a systematic review. Health Psychol Behav Med 2014;2:983-1008.

22. Roche AM, et al. Workplace mental illness and substance use disorders in male-dominated industries: A Systematic Literature Review: National Centre for Education and Training on Addiction (NCETA), 2012

23. Linzer M, Spitzer R, Kroenke K, et al. Gender, quality of life, and mental disorders in primary care: results from the PRIME-MD 1000 study. Am J Med 1996;101:526-33.

24. Andrews G, Issakidis $C$, Carter $G$. Shortfall in mental health service utilisation. Br J Psychiatry 2001;179:417-25.

25. Proudfoot J, Clarke J, Birch MR, et al. Impact of a mobile phone and web program on symptom and functional outcomes for people with mild-to-moderate depression, anxiety and stress: a randomised controlled trial. BMC Psychiatry 2013;13:312.

26. Donker T, Petrie K, Proudfoot J, et al. Smartphones for smarter delivery of mental health programs: a systematic review. J Med Internet Res 2013;15:e247.

27. Christensen H, Griffiths KM, Jorm AF. Delivering interventions for depression by using the internet: randomised controlled trial. BMJ 2004;328:265.

28. Cockayne NL, Glozier N, Naismith SL, et al. Internet-based treatment for older adults with depression and co-morbid cardiovascular disease: protocol for a randomised, double-blind, placebo controlled trial. BMC Psychiatry 2011;11:10.

29. Glozier N, Christensen H, Naismith S, et al. Internet-delivered cognitive behavioural therapy for adults with mild to moderate depression and high cardiovascular disease risks: a randomised attention-controlled trial. PLoS One 2013;8:e59139.

30. Price M, Yuen EK, Goetter EM, et al. mHealth: a mechanism to deliver more accessible, more effective mental health care. Clin Psychol Psychother 2014;21:427-36.

31. Cunningham JA, Gulliver A, Farrer L, et al. Internet interventions for mental health and addictions: current findings and future directions. Curr Psychiatry Rep 2014;16:521.

32. Stratton E, Lampit A, Choi I, et al. Effectiveness of eHealth interventions for reducing mental health conditions in employees: A systematic review and meta-analysis. PLoS One 2017;12:e0189904.

33. World Medical Association. World Medical Association Declaration of Helsinki: ethical principles for medical research involving human subjects. JAMA 2013;310:2191-4. 
34. Chan AW, Tetzlaff JM, Altman DG, et al. SPIRIT 2013 statement: defining standard protocol items for clinical trials. Ann Intern Med 2013;158:200-7.

35. Moher D, Schulz KF, Altman DG. The CONSORT statement: revised recommendations for improving the quality of reports of parallelgroup randomised trials. The Lancet 2001;357:1191-4.

36. Australian Bureau of Statistics, Australian and New Zealand standard industrial classification. Canberra: Australian Bureau of Statistics, 2008.

37. Choi I, Milne DN, Glozier N, et al. Using different Facebook advertisements to recruit men for an online mental health study: Engagement and selection bias. Internet Interv 2017;8:27-34.

38. Hopko DR, Lejuez CW, Ruggiero KJ, et al. Contemporary behavioral activation treatments for depression: procedures, principles, and progress. Clin Psychol Rev 2003;23:699-717.

39. Lejuez CW, Hopko DR, Acierno R, et al. Ten year revision of the brief behavioral activation treatment for depression: revised treatment manual. Behav Modif 2011;35:111-61.

40. Wood AM, Joseph S. The absence of positive psychological (eudemonic) well-being as a risk factor for depression: a ten year cohort study. J Affect Disord 2010;122:213-7.

41. Mazzucchelli T, Kane R, Rees C. Behavioral Activation Treatments for Depression in Adults: A Meta-analysis and Review. Clinical Psychology: Science and Practice 2009;16:383-411.

42. Walpola R. What the Buddha taught. 1967: Revised edition. Bedford: Gordon Fraser Gallery,, 1967.

43. Hofmann SG, Sawyer AT, Witt AA, et al. The effect of mindfulnessbased therapy on anxiety and depression: A meta-analytic review. $J$ Consult Clin Psychol 2010;78:169-83.

44. Wang J. Work stress as a risk factor for major depressive episode(s). Psychol Med 1999;35:865-71.

45. Chiesa A, Serretti A. Mindfulness-based stress reduction for stress management in healthy people: a review and meta-analysis. $J$ Altern Complement Med 2009;15:593-600.

46. Jacobson NS, Martell CR, Dimidjian S. Behavioral Activation Treatment for Depression: Returning to Contextual Roots. Clinical Psychology: Science and Practice 2001;8:255-70.

47. Cribb G, Moulds ML, Carter S. Rumination and Experiential Avoidance in Depression. Behaviour Change 2006;23:165-76.

48. Garnefski N, Teerds J, Kraaij V, et al. Cognitive emotion regulation strategies and depressive symptoms: differences between males and females. Pers Individ Dif 2004;36:267-76.

49. Deady M, Peters $\mathrm{D}$, Lang $\mathrm{H}$, et al. Designing smartphone mental health applications for emergency service workers. Occup Med 2017;67:425-8.

50. Fernandez A, et al. . Development and validation of a prediction algorithm for the onset of common mental disorders in a working population. Australian \& New Zealand Journal of Psychiatry 2017112.

51. Peters D, Deady M, Glozier N, et al. Worker Preferences for a Mental Health App Within Male-Dominated Industries: Participatory Study. JMIR Ment Health 2018:5:e30.

52. Titov N, Dear BF, Johnston L, et al. Improving adherence and clinical outcomes in self-guided internet treatment for anxiety and depression: randomised controlled trial. PLoS One 2013;8:e62873.

53. Matthews M, et al. Jo L, Designing mobile applications to support mental health interventions, in Handbook of Research on User Interface Design and Evaluation for Mobile Technology: IGI, Information Science Reference2008:635-56.

54. Bakker D, Kazantzis N, Rickwood D, et al. Mental Health Smartphone Apps: Review and Evidence-Based Recommendations for Future Developments. JMIR Ment Health 2016;3:e7.

55. Kroenke K, Spitzer RL, Williams JBW. The PHQ-9 - Validity of a brief depression severity measure. Journal of General Internal Medicine 2001:16:606-13.

56. Kroenke K, Spitzer RL, Williams JBW. The PHQ-9. J Gen Intern Med 2001;16:606-13.

57. Bech P. Measuring the dimensions of psychological general wellbeing by the WHO-5. QoL Newsletter 2004;32:15-16.

58. Bech P, Gudex C, Johansen S, Staehr Johansen K. The WHO (Ten) Weil-Being Index: Validation in Diabetes. Psychother Psychosom 1996;65:183-90.

59. Kroenke K, Spitzer RL, Williams JB, et al. Anxiety disorders in primary care: prevalence, impairment, comorbidity, and detection. Ann Intern Med 2007:146:317-25.

60. Connor KM, Davidson JR. Development of a new resilience scale: the Connor-Davidson Resilience Scale (CD-RISC). Depress Anxiety 2003:18:76-82.

61. Kessler RC, Barber C, Beck A, et al. The World Health Organization Health and Work Performance Questionnaire (HPQ). J Occup Environ Med 2003;45:156-74.
62. Andrews D, Nonnecke B, Preece J. Electronic Survey Methodology: A Case Study in Reaching Hard-to-Involve Internet Users. Int J Hum Comput Interact 2003;16:185-210.

63. Spitzer RL, et al. Validation and utility of a self-report version of PRIME-MD - The PHQ primary care study. Jama-Journal of the American Medical Association 1999;282:1737-44.

64. Spitzer RL, Kroenke K, Williams JB. Validation and utility of a selfreport version of PRIME-MD: the PHQ primary care study. Primary Care Evaluation of Mental Disorders. Patient Health Questionnaire. JAMA 1999;282:1737-44.

65. Martin A, Rief W, Klaiberg A, et al. Validity of the Brief Patient Health Questionnaire Mood Scale (PHQ-9) in the general population. Gen Hosp Psychiatry 2006;28:71-7.

66. Kocalevent RD, Hinz A, Brähler E. Standardization of the depression screener patient health questionnaire (PHQ-9) in the general population. Gen Hosp Psychiatry 2013;35:551-5.

67. Kroenke K, Spitzer RL. The PHQ-9: A New Depression Diagnostic and Severity Measure. Psychiatr Ann 2002;32:509-15.

68. Löwe B, Wahl I, Rose M, et al. A 4-item measure of depression and anxiety: validation and standardization of the Patient Health Questionnaire-4 (PHQ-4) in the general population. J Affect Disord 2010;122:86-95.

69. Campbell-Sills L, Stein MB. Psychometric analysis and refinement of the Connor-davidson Resilience Scale (CD-RISC): Validation of a 10-item measure of resilience. J Trauma Stress 2007;20:1019-28.

70. Southwick SM, Vythilingam M, Charney DS. The psychobiology of depression and resilience to stress: implications for prevention and treatment. Annu Rev Clin Psychol 2005;1:255-91.

71. Krieger T, Zimmermann J, Huffziger S, et al. Measuring depression with a well-being index: further evidence for the validity of the WHO Well-Being Index (WHO-5) as a measure of the severity of depression. J Affect Disord 2014;156:240-4.

72. Lecrubier $\mathrm{Y}$, Sheehan DV, Weiller E, et al. The Mini International Neuropsychiatric Interview (MINI). A short diagnostic structured interview: reliability and validity according to the CIDI. European Psychiatry 1997;12:224-31.

73. Kroenke K, Strine TW, Spitzer RL, et al. The PHQ-8 as a measure of current depression in the general population. $J$ Affect Disord 2009;114(1-3):163-73.

74. Imamura K, Kawakami N, Furukawa TA, et al. Effects of an Internetbased cognitive behavioral therapy (iCBT) program in Manga format on improving subthreshold depressive symptoms among healthy workers: a randomized controlled trial. PLoS One 2014;9:e97167. ArtID e97167.

75. Johnson PO, Neyman J. Tests of certain linear hypotheses and their application to some educational problems. Statistical Research Memoires 1936;1:57-93.

76. Tan L, Wang M-J, Modini M, et al. Erratum to: preventing the development of depression at work: a systematic review and metaanalysis of universal interventions in the workplace. BMC Med 2014;12:212

77. Andersson G, Cuijpers P. Internet-Based and Other Computerized Psychological Treatments for Adult Depression: A Meta-Analysis. Cogn Behav Ther 2009;38:196-205.

78. Cuijpers P, Donker T, Johansson R, et al. Self-guided psychological treatment for depressive symptoms: a meta-analysis. PLoS One 2011;6:e21274.

79. Green P, MacLeod CJ. SIMR: an R package for power analysis of generalized linear mixed models by simulation. Methods Ecol Evol 2016:7:493-8.

80. Harvey SB, Modini M, Joyce S, et al. Can work make you mentally ill? A systematic meta-review of work-related risk factors for common mental health problems. Occup Environ Med 2017;74:301-10.

81. Harrison V, Proudfoot J, Wee PP, et al. Mobile mental health: review of the emerging field and proof of concept study. $J$ Ment Health 2011;20:509-24.

82. Martell C.R., Dimidjian S, et al; Behavioral Activation for Depression: A Clinician's Guide: The Guilford Press, 2013.

83. Ly KH, Topooco N, Cederlund H, et al. Smartphone-Supported versus Full Behavioural Activation for Depression: A Randomised Controlled Trial. PLoS One 2015;10:e0126559.

84. Rochlen AB, O'Brien KM. The relation of male gender role conflict and attitudes toward career counseling to interest in and preferences for different career counseling styles. Psychol Men Masc 2002;3:9-21.

85. Atkinson DR, Worthington RL, Dana DM, et al. Etiology beliefs, preferences for counseling orientations, and counseling effectiveness. J Couns Psychol 1991;38:258-64.

86. Leykin $\mathrm{Y}$, Muñoz RF, Contreras $\mathrm{O}$, et al. Results from a trial of an unsupported internet intervention for depressive symptoms. Internet Interv 2014;1:175-81. 
87. Donker T, Bennett K, Bennett A, et al. Internet-delivered interpersonal psychotherapy versus internet-delivered cognitive behavioral therapy for adults with depressive symptoms: randomized controlled noninferiority trial. J Med Internet Res 2013;15:e82.

88. Christensen H, Griffiths K, Groves C, et al. Free range users and one hit wonders: community users of an Internet-based cognitive behaviour therapy program. Aust N Z J Psychiatry 2006;40:59-62.

89. Kroenke K, Spitzer RL, Williams JB. The PHQ-9: validity of a brief depression severity measure. J Gen Intern Med 2001;16:606-13.

90. Newman SC, Shrout PE, Bland RC. The efficiency of two-phase designs in prevalence surveys of mental disorders. Psychol Med 1990;20:183-93.
91. Van Hooff M, McFarlane AC, Davies CE, et al. The Australian Defence Force Mental Health Prevalence and Wellbeing Study: design and methods. Eur J Psychotraumatol 2014;5:23950. epub.

92. Burdett $\mathrm{H}$, Fear NT, Jones N, et al. Use of a two-phase process to identify possible cases of mental ill health in the UK military. Int $J$ Methods Psychiatr Res 2016;25:168-77.

93. Manea L, Gillbody S, McMillan D. Optimal cut-off score for diagnosing depression with the Patient Health Questionnaire (PHQ9): a meta-analysis. CMAJ 2012;184:E191-E196.

94. Pickles A, Dunn G, Vázquez-Barquero JL. Screening for stratification in two-phase ('two-stage') epidemiological surveys. Stat Methods Med Res 1995;4:73-89. 